\title{
Therapie der kleinen Atemwege: Grundlagen und Möglichkeiten
}

\section{Therapy for Small Airways Disease: Basic Principles and Therapeutic Implications}

Autoren

Institute
P. Haidl', C. Kroegel ${ }^{2}$, M. Kohlhäufl ${ }^{3}$, T. Voshaar ${ }^{4}$

${ }^{1}$ Fachkrankenhaus Kloster Grafschaft, Abteilung Pneumologie II, Schmallenberg

${ }^{2}$ Medizinische Klinik I, Abteilung Pneumologie\&Allergologie, Friedrich-Schiller-Universtät, Jena

${ }^{3}$ Klinik Schillerhöhe, Zentrum für Pneumologie und Thoraxchirurgie, Stuttgart-Gerlingen

${ }^{4}$ Medizinische Klinik III, Schwerpunkt Pneumologie, Allergologie, Zentrum für Schlaf- und Beatmungsmedizin, Moers eingereicht 15.8.2011 akzeptiert nach Revision 12. 12.2011

\section{Bibliografie}

Dol http://dx.doi.org/

10.1055/s-0031-1291616

Pneumologie 2012; 66: 356-360

(c) Georg Thieme Verlag KG Stuttgart · New York

ISSN 0934-8387

\section{Korrespondenzadresse} Dr. Peter Haidl

Fachkrankenhaus Kloster Grafschaft

Abteilung Pneumologie II

Annostr. 1

57392 Schmallenberg

p.haidl@t-online.de

\section{Zusammenfassung \\ $\nabla$}

Der Beitrag zeigt die Möglichkeiten auf, die kleinen Atemwege („small airways“) gezielt zu erreichen. Neben der Therapie chronisch obstruktiver Lungenerkrankungen kann dies für die Entwicklung von Behandlungskonzepten unterschiedlicher Bronchiolitiden von großer Bedeutung sein. Kleine Aerosolpartikel, ein langsamer und möglichst kontrollierter Inspirationsfluss und eine Atemanhaltezeit nach Inhalation von 5-10 sec für Steroidpartikel sind essentielle Einflussfaktoren für eine periphere Lungendeposition. Klinische Daten zum Vergleich von Steroiden mit extrafeiner und konventioneller Partikelgröße bei Asthma bronchiale liegen insbesondere aus methodischen Gründen nur begrenzt vor. Extrafeine Steroidaerosole zeigten einen Trend zur Reduktion von Symptomen, zur positiven Beeinflussung von Biomarkern und zur Abnahme der Lungenüberblähung.

\section{Einleitung \\ $\nabla$}

In vorausgegangenen Publikationen in dieser Zeitschrift stellten Husemann et al. die aktuellen lungenfunktionellen Möglichkeiten dar, um eine bevorzugt in den kleinen Atemwegen lokalisierte Lungenerkrankung zu diagnostizieren [1]. Weiterhin gaben Kroegel et al. einen Überblick über Grundlagen und Diagnostik der Bronchiolitiden, d.h. in der Lungenperipherie und damit in den kleinen Atemwegen gelegenen Erkrankungen [2, 3].

Wird die Diagnose einer Erkrankung der kleinen Atemwege („small airways disease“) gestellt, beeinflusst dies dann auch die Art und Weise der Therapie?

Der nachfolgende Beitrag soll dieser Frage nachgehen.

\section{Abstract \\ $\nabla$}

This paper describes the possibility of targeting the small airways. In addition to aiding in the therapy for chronic obstructive lung diseases this may prove to be invaluable in the development of treatment strategies for diseases of the bronchioli. Essential factors in peripheral lung deposition include extra-fine particles, a slow and controlled inspiratory flow and an endexspiratory breathhold of 5-10 sec (especially for steroids). Due to methodological difficulties, clinical data comparing steroids with larger or extra-fine particles are limited in the field of asthma therapy. However, research suggests a trend for reduced symptoms, positively affected biomarkers and decreased lung hyperinflation when steroids with extrafine particles are used.

\section{Ist „Targeting“ der kleinen Atemwege möglich?}

Die inhalative Applikation von Medikamenten besitzt eine große therapeutische Breite. Allerdings ist die in der Lunge deponierte Wirkstoffmenge großen Schwankungen unterworfen. Wichtige praxisrelevante Parameter sind insbesondere die Partikelgröße des Aerosols sowie der Inspirationsfluss.

Bei einem Dosieraerosol (pressurized metered dose inhaler $=$ pMDI) ist die Partikelgröße des im Treibgas suspendierten Pulvers vorgegeben. Die Deposition kann durch den Patienten verbessert werden, wenn dieser einen möglichst langsamen Inspirationsfluss generiert. Dadurch sinkt die in der Regel mit 70 bis $80 \%$ sehr hohe oropharyngeale Abscheiderate und die Lungendeposition steigt $[4,5]$. Durch die Verwendung eines Spacers (vorgeschaltetes großvolumiges Hohlraumsys- 


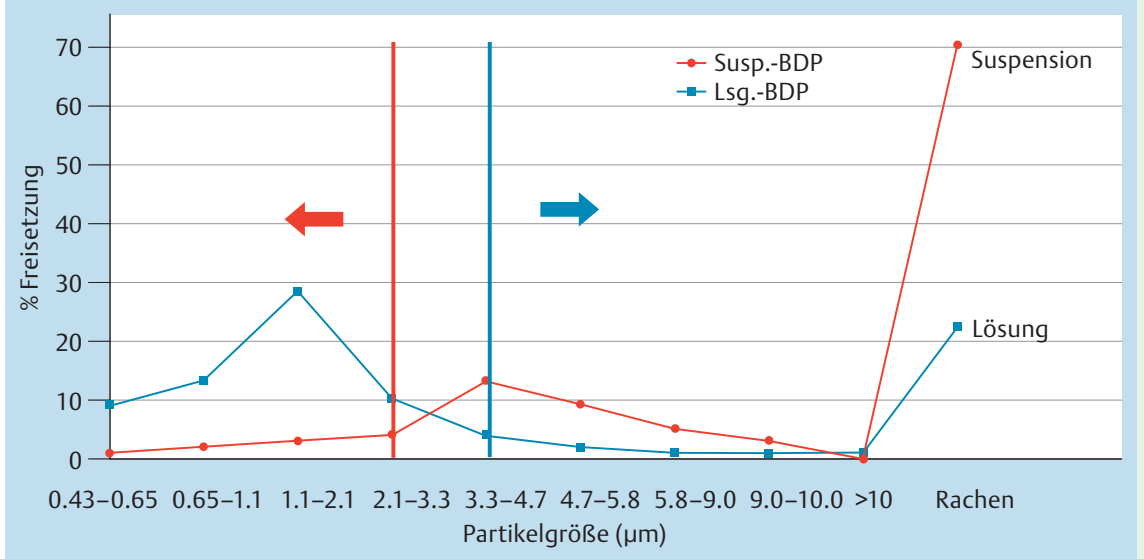

Abb. 1 Partikelgrößenverteilung von BDP in Suspension und Lösung, gemessen in vitro mit einem Impactor. Bei der Suspension weist die rote Linie auf den Anteil der Partikel kleiner als 2,1 $\mu \mathrm{m}$ hin, bei der Lösung weist die blaue Linie auf den Anteil größer als $3,3 \mu \mathrm{m}$ hin.

tem) sinkt die oropharyngeale Deposition bis auf Werte unter $10 \%$, da die rasche Freisetzung der anfangs noch vorhandenen Treibgaströpfchen nicht im Oropharynx mit Anprall an die Rachenhinterwand, sondern in dem deutlich größeren Spacer stattfindet. Das Treibgas verdampft und die Partikel kommen zur Ruhe. Aus dem Spacer können sie dann mit einem langsamen Fluss inhaliert werden.

Bei den inhalativen Steroiden stehen seit einigen Jahren pMDI mit unterschiedlicher Partikelgröße zur Verfügung. Je kleiner die Partikel sind, desto weiter dringen diese in die Lungenperipherie vor [6]. Allerdings sind therapeutische Aerosole polydispers. Das heißt, es gibt eine weite Streuung der Partikelgröße um den Median ( $\bullet$ abb. 1). Ein pMDI mit einem $\operatorname{MMAD}($ „mass median aerodynamic diameter") von $4 \mu \mathrm{m}$ enthält in einem Prozentsatz von ca. $15 \%$ auch feine Partikel unter $2 \mu \mathrm{m}$. Umgekehrt werden mit einem „extrafeinen“ pMDI (MMAD 1.30 £0.1) [7] 15\% Partikel über $3 \mu \mathrm{m}$ abgegeben. Prinzipiell wäre die Verwendung monodisperser Partikel für das Targeting wirkungsvoller. Mit kleineren Dosen könnte der gleiche klinische Effekt erreicht werden, allerdings würden die Produktionskosten erheblich steigen. Bei den Trockenpulverinhalatoren (dry powder inhaler=DPI) wird die Partikelgröße des Pulvers zusätzlich durch den Inspirationsfluss des Patienten und den Widerstand des Inhalationssystems bestimmt, da das Pulver verklumpt vorliegt und erst desagglomeriert werden muss. Dadurch ist die Höhe der Deposition schwerer vorhersehbar und wesentlich vom Ausmaß einer möglicherweise vorhandenen Bronchialobstruktion abhängig [8,9]. Im Gegensatz zu einem pMDI muss der Inspirationsfluss hoch sein, da diese Kraft zur Desagglomeration der Pulverpartikel notwendig ist. Die Tab. 1 zeigt Partikelgrößen für pMDI's [7] und verschiedene DPI's [10 - 13] bei optimalem Atemmanöver. Diese Werte können aber nur grobe Annäherungen sein.

Wenige Daten liegen zur Partikeldeposition bei Patienten mit schwerer Bronchialobstruktion vor. In einer aktuellen Untersuchung verglich DeBacker [7] die Lungendeposition eines extrafeinen Aerosols (Formoterol/Beclometason) bei Gesunden und Patienten mit schwerer COPD (FEV1 43.6\% Soll). Das Aerosol wurde radioaktiv markiert und mittels Gamma-Kamera die Deposition in der gesamten Lunge sowie die regionale Verteilung (Verhältnis der zentralen zur peripheren Aktivität= Penetrationsindex) gemessen. Die Gesamtdeposition in der Lunge betrug 34\% bei den Gesunden und 33\% in der COPD-Gruppe. Auch die Standardabweichung (9\%) unterschied sich nicht. Dieses überraschte eigentlich nicht, denn beide Gruppen atmen etwa das gleiche Atemzugvolumen. Allerdings verschiebt sich der Penetrationsindex von 1.42 bei Gesunden auf 1.94 bei den COPD-Patien-
Tab. 1 Vergleich des mittleren aerodynamischen Massendurchmessers $(\mu \mathrm{m})$ verschiedener Inhalationssysteme.

\begin{tabular}{|ll|}
\hline Budesonid Turbohaler (Inspir. fluss 60I/min) & 2.9 \\
\hline Budesonid pMDI (Suspension) & 3.5 \\
\hline Fluticason Diskus (Inspir. fluss 60I/min) & 3.5 \\
\hline Fluticason pMDI (Suspension) & 2.8 \\
\hline Beclomethason pMDI (Lösungsaerosol) & 1.3 \\
\hline Ciclesondie pMDI (Lösungsaerosol) & 1.9 \\
\hline
\end{tabular}

$\mathrm{pMDI}=$ pressurized metered dose inhaler $[7,10-13]$

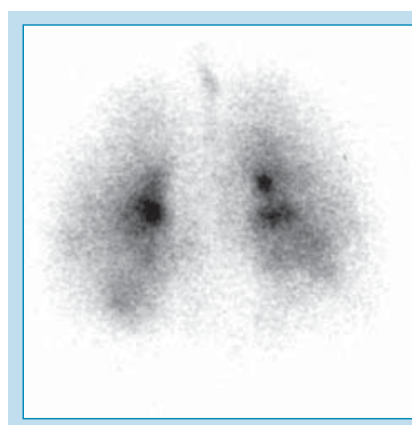

Chronisch obstruktive Bronchitis

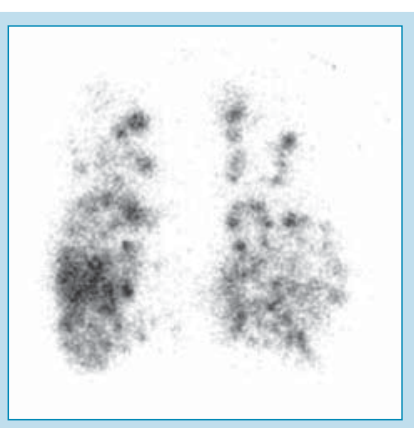

Lungenemphysem
Abb. 2 Ventilationsszintigramme bei chronisch obstruktiven Lungenerkrankungen. Verwendet wurde ein Vernebler mit einem Spacer. Die erzeugte Partikelgröße liegt um $2 \mu \mathrm{m}$.

ten, d.h. die Deposition verschiebt sich nach zentral. Dies bedeutet, dass eine mögliche Zielzone bei peripherer Erkrankung mit zunehmender Obstruktion schlechter erreicht wird. Würde ein Aerosol mit einer „konventionellen“ Partikelverteilung um 3-4 $\mu \mathrm{m}$ verwendet, käme es zu einem weiteren Dosisverlust in der Peripherie. Eindrucksvoll sind Ventilationsszintigramme ( $\triangle$ Abb.2) bei Verwendung von polydispersen, von einem Vernebler erzeugten Aerosolen (MMAD um $2 \mu \mathrm{m}$ ) bei Patienten mit einem Lungenemphysem. In diesem „Leopardenmuster“ ist erkennbar, dass größere Emphysemareale einer Aerosoltherapie nicht mehr zugänglich sind. In diesen destruierten Arealen würden auch keine Therapieeffekte mehr zu erwarten sein. Auf der anderen Seite ermöglichen allein extrafeine Partikel überhaupt noch eine Wirkstoffbelegung der weniger betroffenen Lungenanteile.

Eine aktuelle Studie [13] wies auf die besondere Bedeutung der Atemanhaltezeit nach erfolgter Inhalation bei extrafeinen Steroidaerosolen hin. Eine Zeitspanne von $10 \mathrm{sec}$ führte zu einer 
Lungendeposition von $60 \pm 14 \%$. Eine kurze Atemanhaltezeit von nur einer Sekunde senkte die Lungendeposition auf $44 \pm 11 \%$. Das gilt bevorzugt für Steroidaerosole, da diese trotz der hohen Feuchte im Bronchialsystem infolge ihrer Lipophilie kein Wasser aufnehmen und nicht wachsen. Hygroskopische Partikel, wie Beta-Mimetika, werden durch Wachstum 2-3-fach größer und deponieren damit schon während der Inspirationsphase, sodass kaum noch Partikel exhaliert werden.

Zusammenfassend kann über die Partikelgröße und den Atemfluss der Depositionsort in der Lunge beeinflusst werden. pMDI ggfs. in Verbindung mit Spacern haben methodische Vorteile, wenn die peripheren Atemwege erreicht werden sollen. $\mathrm{Zu} \mathrm{Be-}$ denken ist ferner, dass mit zunehmender Obstruktion und damit Schwere der Erkrankung die Wirkstoffdeposition in Richtung zentrales Bronchialsystem verschoben wird. Zusätzlich können kleine Inspirationsvolumina die Lungendeposition weiter reduzieren. Damit kann ein relevanter therapeutischer Effekt in den kleinen Atemwegen nur über kleine Partikel, einen langsamen Atemfluss, eine ausreichende Inspirationstiefe und eine längere Atemanhaltezeit erzielt werden. Außerdem sind pMDI bei schwerer Obstruktion den DPI überlegen, da bei DPI im Einzelfall der Inspirationsfluss keine ausreichende lungengängige Dosis erlaubt. Es gibt eine hohe Variabilität der Inspirationsflüsse. Flüsse über $30 \mathrm{~L} / \mathrm{min}$ (am Mund bzw. distal der Stenose des Inhalationssystems), die für eine akzeptable Lungendeposition erforderlich sind, können von Patienten mit COPD und zystischer Fibrose erreicht werden, aber nicht von allen $[14,15]$.

\section{Therapeutische Effekte extrafeiner Formulierungen} $\nabla$

Generell führen kleine Partikel zu einer höheren Lungendeposition, die sich in der klinischen Wirksamkeit niederschlägt. Daher sind $200 \mu \mathrm{g}$ Beclometason extrafeine Partikel (MMAD ca. 1 - $2 \mu \mathrm{m}$ )

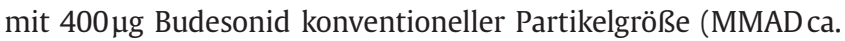
$3-4 \mu \mathrm{m}$ ) sowie $400 \mu \mathrm{g}$ Beclomethason und $500 \mu \mathrm{g}$ Fluticason in der klinischen Wirkung vergleichbar.

Bei stabilem Asthma wurde eine extrafeine Fixkombination sowohl mit Fixkombinationen, appliziert mit dem Turbohaler [16], als auch mit dem Diskus [17] verglichen. Bei diesen „Non Inferiority“ Studien konnten äquivalente Wirkungen gezeigt werden. Ziel war es hier, die Gleichwertigkeit zu belegen. Die Fallzahlen waren nicht berechnet, um ggfs. eine signifikante Überlegenheit nachzuweisen.

In diesen Studienkollektiven war das Ausmaß der Obstruktion gering und mögliche Unterschiede im Schweregrad einer peripheren Obstruktion nicht erfasst. Die stärkere Entzündung in den kleinen Atemwegen („small airways disease“) hätte wahrscheinlich zu einem schwerer therapierbaren Asthma geführt und die Patienten hätten dann die Einschlusskriterien nicht erfüllt.

Daher sind Untersuchungen an Patienten mit schwer kontrollierbarem Asthma für die Abschätzung des Therapieeffektes in der Lungenperipherie von besonderer Bedeutung.

Thongngarm et al. [18] schlossen 30 Patienten mit mäßig kontrolliertem Asthma (mehr als 2 Hübe kurzwirksames Beta-Mimetikum pro Tag, nächtliche Luftnot mindestens einmal wöchentlich) trotz ausreichend dosierter inhalativer Steroidmedikation ein und verglichen HFA-Beclometason (extrafeine Partikel) mit Fluticason (konventionelle Partikelgröße) jeweils im pMDI. Zielgrößen als Marker der peripheren Erkrankung waren das Closing volume und das FEF 25 - 75. HFA-Beclometason erzielte eine sig- nifikante Verbesserung dieser Parameter. Zusätzlich sank der Verbrauch der Bedarfsmedikation signifikant.

Ohbayashi et al. [19] verglichen ebenfalls HFA-Beclometason (pMDI) mit Fluticason (Diskus) und untersuchten über 6 Monate in einem cross-over Design den Einfluss auf die Eosinophilen und das eosinophile kationische Protein im Serum sowie im induzierten Sputum. Das HFA-Beclometason senkte die Zahl der Eosinophilen im Serum als auch im Sputum. Die im Normbereich liegenden Lungenfunktionsparameter zeigten keine Änderung. Der Symptomscore besserte sich ebenso wie die Einschränkung der körperlichen Aktivität nach dem Wechsel von Fluticason auf HFA-BDP.

Scichilone et al. [20] wählten Veränderungen im MethacholinProvokationstest und den Stickstoff-Auswaschtest als primäre Endpunkte. Je 15 Patienten mit moderatem Asthma inhalierten täglich über 3 Monate entweder extrafeines Beclometason/Formoterol oder Fluticason/Salmeterol. Der Anstieg der Methacholin-Schwellendosis war in der Beclometason/Formoterol-Gruppe signifikant größer. Kenngrößen des Stickstoff-Auswaschtests wie Closing capacity oder die Steigung der Auswaschkurve in Phase III zeigten hingegen keine signifikanten Unterschiede.

Ein anderer Ansatz wurde von Goldin et al. [21] verfolgt. Verglichen wurden 44 Patienten mit Asthma, die entweder HFA-Beclometason (MMAD 1.0 $\mu \mathrm{m}$ ) oder CFC-Beclometason (MMAD 3.8 $\mu \mathrm{m}$ ) über 4 Wochen inhalierten. Im HRCT wurde die Verteilung der Lungendichte vor und nach einer Methacholin-Provokation als auch nach dem Therapieintervall bestimmt. Obwohl sich Symptome und spirometrische Daten nicht änderten, stieg die Lungendichte als Zeichen der Abnahme des „air-trapping“ in der HFABeclometason-Gruppe signifikant an.

Bisher liegen nur wenige klinische Daten vor, um die Rolle von Steroiden mit extrafeiner Partikelgröße bei „small airways disease“ beurteilen zu können. Diese Situation beruht auch auf dem Problem der Auswahl eines Patientenkollektivs mit führender Manifestation der Erkrankung in den peripheren Atemwegen sowie dem geeigneten primären Endpunkt. Es darf nicht vergessen werden, dass Inhalationssysteme mit einem MMADvon 3-4 $\mu \mathrm{m}$ aufgrund der polydispersen Verteilung die Lungenperipherie ebenfalls erreichen, wenn auch weniger verlässlich und mit deutlich geringeren Dosen. Dies mag bei einem stabilen Asthma ohne persistierende Obstruktion bereits ausreichen, eine periphere Entzündung zu kontrollieren.

\section{Generelle Vorteile extrafeiner Aerosolpartikel $\nabla$}

Wie oben dargestellt, erreichen extrafeine Aerosolpartikel die peripheren Atemwege. Es existieren zusätzliche praktisch bedeutsame Vorteile, auf die nachfolgend eingegangen werden soll. 1. Es gibt eine Reihe von Patienten mit Asthma, die beruflich oder in ihrer Freizeit auf ihre Stimme angewiesen ist. Diese ist besorgt bezüglich einer möglichen Heiserkeit infolge einer Steroidinhalation. Die oropharygeale Deposition liegt für DPI und MDI mit einem MMAD von 3-4 $\mu$ m bei $70-80 \%$. Extrafeine Partikel reduzieren die Mund-Rachen-Deposition auf ca. $30 \%-40 \%$ und senken damit die Nebenwirkungsrate relevant. Die wirksamste Maßnahme zur Vermeidung der Komplikationen Soor und Stimmbanddysfunktion ist die zusätzliche Verwendung eines Spacers bei den extrafeinen Partikeln, der die oropharyngeale Deposition auf ca. $4 \%$ senkt [13].

2. Eine nuklearmedizinische Untersuchung von Leach et al. [22] zeigte einen weiteren Vorzug bei der Anwendung von pMDI. 
Bekannt ist das Koordinationsproblem. Kleine Partikel zeigen bei suboptimaler Inhalationstechnik nur eine moderate Reduktion der Lungendeposition im Vergleich zu konventionellen MDIs. Dies bedeutet, dass extrafeine Partikel die negativen Folgen einer insuffizienten Koordination von Inhalationsbeginn und Sprühstossauslösung vermindern.

3. Extrafeine Partikel sind bei schwerer Bronchialobstruktion von Bedeutung, um überhaupt eine therapeutisch wirksame Lungendeposition in den mittelgroßen und kleinen Atemwegen (Generation 6-16) zu erzielen. Partikel mit einem MMAD von 3-5 $\mu \mathrm{m}$ werden im Zusammenhang mit der Obstruktion und zentralen Turbulenzen häufig bereits an den Segment- oder Subsegmentcarinen abgeschieden [23].

4. Ein weiterer Aspekt ist neben der Therapie obstruktiver Lungenerkrankungen die mögliche zukünftige Behandlung von Bronchiolitiden. Auf die Vielfalt und klinische Bedeutung hat Kroegel [2] in seinem Übersichtsbeitrag hingewiesen. Ein inhalativer Therapieansatz kann nur mit geeigneter Partikelgröße in Verbindung mit einem moderaten Inspirationsfluss erfolgsversprechend sein. Zur Verfügung stehen hierzu bereits Inhalationssysteme (z.B. AKITA ${ }^{\circledR}$ ), die computergestützt den Atemfluss kontrollieren und Vernebler mit kleinen Partikeln (MMAD um $2 \mu \mathrm{m}$ ) verwenden [24].

\section{Limitationen}

\section{$\nabla$}

Je kleiner Aerosolpartikel sind, desto kleiner wird das Volumen, das mit der transportierten Medikamentendosis korreliert. Die Dosis nimmt in der dritten Potenz im Vergleich zur Partikelgröße ab. Beispielsweise reduziert sich die Dosis um das 8-fache, wenn die Partikelgröße halbiert wird. Dies muss durch eine höhere Dichte des Aerosols ausgeglichen werden.

Bei Beta-Mimetika gibt es Untersuchungen, dass eine Steigerung des Anteils extrafeiner Partikel die Nebenwirkungsrate (Senkung des Serum-Kaliumspiegels), nicht aber den bronchodilatatorischen Effekt erhöht [25]. Somit kann im Einzelfall die separate Gabe eines Steroids mit extrafeiner Formulierung und eines Beta-Mimetikums mit konventioneller Partikelgröße sinnvoll sein. Den Autoren ist bewusst, dass Fragen zur klinischen Bedeutung der Inhalation extrafeiner Partikel nicht vollständig beantwortet werden können. Es wäre erfreulich, wenn der Beitrag als Diskussionsgrundlage dienen könnte, sich auch in Deutschland intensiver mit der Thematik und der Relevanz einer „zielgenauen“ Therapie $z u$ beschäftigen.

\section{Zusammenfassung}

Targeting der peripheren Atemwege ist möglich. Neben der Therapie chronisch obstruktiver Lungenerkrankungen kann dies für die Entwicklung von Behandlungskonzepten der unterschiedlichen Bronchiolitiden wichtig sein.

Kleine Aerosolpartikel, ein langsamer und möglichst kontrollierter Inspirationsfluss und eine Atemanhaltezeit nach Inhalation von 5-10 sec für Steroidpartikel sind essentielle Einflussfaktoren für eine periphere Lungendeposition.

Bisher liegen klinische Daten zum Vergleich von Steroiden mit extrafeiner und konventioneller Partikelgröße bei Asthma bronchiale u.a. aus methodischen Gründen nur begrenzt vor. Ein Trend zur Symptomsenkung, der positiven Beeinflussung von
Biomarkern und zur Abnahme der Lungenüberblähung zeichnet sich ab.

\section{Interessenkonflikt \\ $\nabla$}

Die Autoren sind Mitglieder der DESA (Deutsche Expertengruppe Small airways) ebenso wie Dr. Dr. Joachim Körner, Manuela Kulle, Dr. Detlef Nachtigall, Dr. Astrid Riebeling und Prof. Dr. Andreas Schmitt, alle Mitarbeiter der Fa. Chiesi Hamburg. P. Haidl, M. Kohlhäufl und T. Voshaar haben Vortragshonorare von diversen Herstellern von Inhalationssystemen erhalten.

\section{Literatur}

1 Husemann K, Haidl P, Kroegel C et al. Diagnostik und Therapie der kleinen Atemwege (Teil I): Lungenfunktionsanalyse. Pneumologie 2012; 66: $283-289$

2 Kroegel C, Haidl P, Kohlhäufl M et al. Bronchiolitis Teil 1 - Anatomische Grundlagen, Klassifikation, klinische Präsentation und Bildgebung. Pneumologie 2012; 66: 28-38

3 Kroegel C, Haidl P, Kohlhäufl M et al. Bronchiolitis Teil 2 - Diagnostischer Zugang und Therapie. Pneumologie 2012; 66: 78 -88

4 Leach CL, Davidson PJ, Bruce E et al. Lung deposition of hydrofluoroalkane-134a beclomethasone is greater than that of chlorofluorocarbon fluticasone and chlorofluorocarbon beclomethasone. Chest 2002; 122 : 510-516

5 Dolovich M, Eng P. Rhem R. Impact of oropharyngeal deposition on inhaled dose. J Aerosol Med 1998; 11 (Suppl 1): 112-115

6 Usmani OS, Biddiscombe MF, Barnes PJ. Regional lung deposition and bronchodilator response as a function of beta2-agonist particle size. Am J Respir Crit Care Med 2005; 172: 1497-1504

7 DeBacker W, Devolder A, Poli G et al. Lung deposition of BDP/formoterol HFA pMDI in healthy volunteers, asthmatic, and COPD patients. J Aerosol Med Pulmon Drug Del 2010; 23: 137-148

8 Köhler D. Wirksamkeit und Akzeptanz von Pulverinhalatoren. Dtsch med Wschr 1995; 120: $1401-1404$

9 Kohlhäufl M, Haidl P, Voshaar T et al. Pulverinhalationssysteme. Dtsch Med Wochenschr 2004; 129: 2048-2052

10 Fabbri LM, Nicolini G, Olivieri D et al. Inhaled beclomethasone diproprionate/formoterol extra-fine combination in the treatment of asthma: evidence and future perspectives. Expert Opin Pharmacother 2008; 9: 479-490

11 De Vries TW, Rottier BL, Gjaltema D et al. Comparative in vitro evaluation of four corticosteroid metered dose inhalers: consistency of delivered dose and particle size distribution. Respir Med 2009; 103: $1167-1173$

12 Feddah MR, Brown KF, Gipps EM et al. In-vitro characterization of metered dose inhaler versus dry powder inhaler glucocorticoid products: Influence of inspiratory flow rates. J Pharm Pharmaceut Sci 2000; 3: 317-324

13 Leach CL, Colice GL. A pilot study to assess lung deposition of HFA-beclomethasone and CFC-Beclomethasone from a pressurized metered dose inhaler with and without add-on spacers and using varying breathhold times. J Aerosol Med Pulm Drug Delivery 2010; 23: 355 361

14 Borgström L. On the use of dry powder inhalers in situations perceived as constrained. J Aerosol Med 2001; 14: $281-287$

15 Tiddens HA, Geller DE, Challoner P et al. Effect of dry powder inhaler resistance on the inspiratory flow rates and volumes of cystic fibrosis patients of six years and older. J Aerosol Med 2006; 19: 456-465

16 Papi A, Paggiaro PL, Nicolini $G$ et al. Inhaled combination asthma treatment versus symbicort (ICAT SY) Study group: beclomethasone/formoterol versus budesonide/formoterol combination therapy in asthma. Eur Respir J 2007; 29: 682 -689

17 Papi A, Paggiaro PL, Nicolini G et al. ICAT SE study group: beclomethasone/formoterol versus fluticasone/salmeterol inhaled combination in moderate to severe asthma. Allergy 2007; 62: $1182-1188$

18 Thongngarm T, Silkoff PE, Kossack WS et al. Hydrofluoroalkane-134a beclomethasone or chlorofluorocarbon fluticasone: effect on small airways in poorly controlled asthma. Journal of Asthma 2005; 42: $257-$ 263 
19 Ohbayashi H, Adachi M. HFA-Beclomethasone diproprionate effectivly improves airway eosinophilic inflammation including the distal airways of patients with mild to moderate asthma as compared with fluticasone proprionate in a randomized open double-cross study. Allergology International 2008; 57: 1 -8

20 Scichilone N, Battaglia S, Sorino C et al. Effects of extra-fine inhaled beclomethasone/formoterol on both large and small airways in asthma. Allergy 2010; 65: 897-902

21 Goldin JG, Tashkin DP, Kleerup EC et al. Comparative effects of HFA and CFC beclomethasone diproprionate inhalation on small airways: assessment with functional helical thinsection computed tomography. J Allergy Clin Immunol 1999; 104: 258-267
22 Leach CL, Davidson PJ, Hasselquist BE et al. Influence of particle size and patient dosing technique on lung deposition of HFA-beclomethasone from a metered dose inhaler. J Aerosol Med 2005; 18: 379-385

23 Köhler D, Fleischer $W$. Was ist gesichert in der Inhalationstherapie? München: Arcis; 1988

24 Fischer A, Stegemann J, Scheuch $G$ et al. Novel devices for individualized controlled inhalation can optimize aerosol therapy in efficacy, patient care and power of clinical trials. Eur J Med Res 2009; 14 (Suppl 4): 71 77

25 Weda $M$, Zanen $P$, de Boer $A H$ et al. An investigation into the predictive value of cascade impactor results for side effects of inhaled salbutamol. Int J Pharm 2004; 287: $79-87$ 